\title{
Antimicrobial Properties of Catha edulis (Miraa) against Select Bacterial and Fungal Pathogens, an in-vitro Experimental Study
}

Dennis Kithinji ( $\nabla$ dennohkithinji@gmail.com )

University of Nairobi

\section{Susan Maina}

University of Nairobi

Stanley Ndwigah

University of Nairobi

Hannington Mugo

University of Nairobi

Julius Oyugi

University of Nairobi Institute of Tropical Medicine and Infectious Diseases

\section{Research Article}

Keywords: Catha edulis, Miraa, Antimicrobial, Extracts, S. aureus, MRSA, S. pyogenes, S. pneumoniae, E. coli, C. albicans

Posted Date: March 30th, 2021

DOl: https://doi.org/10.21203/rs.3.rs-293264/v1

License: (1) This work is licensed under a Creative Commons Attribution 4.0 International License. Read Full License 


\section{Abstract}

Background: The increasing risk of emergence of antimicrobial resistance can be addressed by discovering alternatives to antibiotics such as plant-based botanicals. In the present study, the antimicrobial properties of aqueous and methanolic extracts of Catha edulis (Miraa) were tested on select pathogenic bacteria and fungi.

Methods: Antimicrobial susceptibility tests were conducted in-vitro using the agar well diffusion method. The aqueous and methanolic extracts were dissolved in water to form $1000 \mathrm{mg} / \mathrm{ml}, 100 \mathrm{mg} / \mathrm{ml}$, and 10 $\mathrm{mg} / \mathrm{ml}$ doses. The antimicrobial susceptibility testing was done in appropriate culture media and conditions. Diameters of zones of inhibition were obtained, their means calculated, and t-tests applied to test significance of differences between means.

Results: The aqueous Miraa extracts at all three concentrations significantly inhibited the growth of all bacterial pathogens except $E$. coli but did not have an effect on $C$. albicans. The largest zones of inhibition for the aqueous extracts were observed at $1000 \mathrm{mg} / \mathrm{ml}$ against $S$. pneumoniae $(28.41 \mathrm{~mm}), S$. pyogenes ATCC 19615 (24.27 mm), MRSA (21.86 mm), and S. aureus clinical isolate $(20.38 \mathrm{~mm})$. Similarly, the largest zones of inhibition for the methanolic extracts were at $1000 \mathrm{mg} / \mathrm{ml}$ against $S$. pneumoniae clinical isolate $(26.75 \mathrm{~mm})$, S. pyogenes ATCC $19615(25.38 \mathrm{~mm})$, S. aureus clinical isolate (19.71 mm), and MRSA ATCC $43300(16.38 \mathrm{~mm})$.

Conclusions: Crude Miraa extracts have significant antimicrobial effects in vitro against the tested microorganisms. Further studies on Miraa extracts to identify the active phytochemicals and investigate their therapeutic effects in-vivo in animal models are indicated.

\section{Background}

The burden of infectious diseases remains a significant threat to health, especially in developing countries. Infections affect a large proportion of the world population. For example, about 55 million people have a urinary tract infection at any given time [1]. Approximately 357 million people get sexually transmitted infections annually [2]. Respiratory, gastrointestinal, and wound infections, as well as bacteremia and sepsis, are also a significant cause of morbidity disability, and mortality.

Infectious diseases are still a substantial problem despite the rapidly increasing use of antibiotics. Between 2000 and 2020, the defined daily doses (DDD) of antibiotics consumed globally increased from 21.1 billion to 34.8 billion, which translates to a $65 \%$ increase [3]. The high rates of use of antibiotics are causing the emergence of antibiotic-resistant bacteria. Besides, synthetic antibiotics are associated with several negative features such as short half-life in vivo, toxicity, and high cost of synthesis [4].

There is a need to reduce the use of antibiotics in response to the problem of antimicrobial resistance (AMR) so that the currently available antibiotics stock may not get depleted [5]. Plant-based botanicals are a viable alternative to antibiotics in efforts to address AMR [6]. Their antimicrobial and 
chemosensitizer effects can be leveraged to optimize the use of the available antibiotics while reducing the pressure on them [7]. Investigating plants used in traditional medicine to treat infectious diseases for antimicrobial effects can provide evidence of their value in managing infectious diseases.

The varieties of $C$. edulis in Yemen, South Africa, Saudi Arabia, and Lebanon have been shown to have antibiotic effects against various bacteria in in-vitro studies [8-12]. Fatima et al. [8] tested methanol, dimethyl sulfoxide (DMSO), and water extracts of Catha edulis Forsk in Saudi Arabia against Staphylococcus aureus, Streptococcus pyogenes, Klebsiella pneumoniae, Escherichia coli, Pseudomonas aeruginosa, Proteus mirabilis, and Candida albicans clinical isolates. The methanol and DMSO extracts had significant zones of inhibition in all the bacteria tested while the aqueous extract was only active against gram positive organisms, particularly Staphylococcus aureus [8]

Siddiqui [11] tested methanolic crude extracts of $C$. edulis purchased from a shop in London against various laboratory-stocked microbes (Escherichia coli, Bacillus magaterium, Brevundimonas diminuta, and Micrococcus luteus) using the antibiotic disc diffusion assay. The researcher established that the extracts had significant (breakpoint of $14 \mathrm{~mm}$ ) antimicrobial effect against all the bacteria tested (zones of inhibition $>16 \mathrm{~mm}$ for $B$. magaterium; $>19 \mathrm{~mm}$ for $M$. luteus) except $E$. coli (zone of inhibition $<11 \mathrm{~mm}$ ) [11]. The variation in E. coli results in the studies by Fatima et al. [8] and Siddiqui [11] imply that Catha edulis grown in different geographical areas could be having varying antimicrobial effects.

Al-hebshi, Al-haroni, and Skaug [9] evaluated the antimicrobial effect of aqueous extracts of Yemen's $C$. edulis against organisms comprising oral microbiota. The extracts showed more effect on the gramnegative bacteria (Porphyromonas gingivalis, Fusobacterium nucleatum, and Prevotella intermedia), which are mainly pathogenic in the mouth, compared to the gram-positive bacteria (Streptococci and Actinomyces), which are mostly the normal flora of the oral cavity [9]. Therefore, Catha edulis could be selectively active against pathogens by sparing normal flora.

Miraa, the common name for Catha edulis varieties cultivated in the Igembe region of Meru County in Kenya, is one of the plants used to treat infectious diseases in traditional medicine. Miraa plant is a dicotyledonous shrub in the Celastraceae family whose twigs are harvested and commonly chewed for recreational purposes [13]. There are $C$. edulis varieties in other parts of the world including Yemen, Ethiopia, Saudi Arabia, South Africa, and Lebanon. According to an ethnobiology study by Kiunga et al. [14], herbalists in Meru use decoctions of the leaves and roots of Miraa to treat oral, respiratory, diarrheal, and urogenital diseases. Miraa can be a suitable source of plant-based botanicals if its antimicrobial properties are established. Its availability is assured given that it is a cultivatable plant without scarcity challenges faced when wild plants are used as sources [15].

The illnesses that herbalists treat using Miraa could be caused by myriad pathogens. $S$. aureus is a common cause of respiratory, urinary tract, and gastrointestinal infections [16], which herbalists apply Miraa decoctions to treat. Methicillin-resistant $S$. aureus (MRSA) is rapidly spreading globally amidst the reducing antibiotic options to treat its infections [17]. Streptococcus pyogenes is a common cause of sore throat, one of the respiratory infections that herbalists treat using Miraa decoctions. Its infections have 
increased in the last three decades and it is commonly developing resistance to antibiotics [18]. Streptococcus pneumoniae, which causes respiratory infections, is a pathogen against which antibiotic resistance is rapidly emerging due to antibiotic selection pressure [19]. Escherichia coli is a common cause of urinary tract, gastrointestinal, and respiratory infections; it is common in outbreaks [20]. Hence, $E$. coli is a suitable representative of gram-negative bacteria in studies to identify plants that can be sources of antimicrobial botanicals. Candida albicans is a fungus that causes oropharyngeal and vulvovaginal candidiasis. Its multi-drug resistant strains are rapidly emerging. Thus, it is an appropriate representative of pathogenic fungi in this study [21].

Our comprehensive review of the literature did not find any study investigating the antimicrobial properties of Miraa, the $C$. edulis cultivated in Kenya. Studying Miraa to determine its antimicrobial effects was essential to generate information on whether it has antimicrobial effects like the varieties tested in other countries. This article reports the in-vitro antimicrobial effects of aqueous and methanolic crude Miraa extracts against Staphylococcus aureus, Streptococcus pneumoniae, Escherichia coli, and Candida albicans clinical isolates, and Streptococcus pyogenes ATCC 19615 and methicillin-resistant Staphylococcus aureus (MRSA) ATCC 43300 standard strains.

\section{Materials And Methods}

\section{Aim, Design and Setting}

The aim of this study was to investigate the antimicrobial effects of Miraa in efforts to explore it as a potential source of plant-based antimicrobial botanicals. An in-vitro experimental study to test the antimicrobial effects of $1000 \mathrm{mg} / \mathrm{ml}, 100 \mathrm{mg} / \mathrm{ml}$, and $10 \mathrm{mg} / \mathrm{ml}$ of Miraa's aquous and methanolic crude extracts on S. aureus, S. pneumoniae, E. coli, and C. albicans clinical isolates, and S. pyogenes ATCC 19615 and MRSA ATCC 43300 standard strains was conducted. The experiments were done in the Drug Analysis and Research Unit (DARU) laboratories in the University of Nairobi, Kenya.

\section{Plant Materials}

Miraa twigs and leaves weighing one kilogram were plucked from Miraa plants in an organic farm in Ithanja village ( $\left.{ }^{\circ} 15^{\prime} 45.0^{\prime \prime} \mathrm{N} 37^{\circ} 56^{\prime} 31.6^{\prime \prime} \mathrm{E}\right)$ in Njia location, Igembe Central District, Meru County, Kenya. They were transported in a Cooler Ice Box at $2-8^{\circ} \mathrm{C}$ to the Drug Analysis and Research Unit on the same day and stored at $-20^{\circ} \mathrm{C}$ until the day of the extraction. A specimen of the twigs and leaves was deposited in the University of Nairobi's School of Biological Science herbarium. It was assigned voucher number DK2020/001. The twigs and leaves were chopped into small pieces using a scissor and ground using a blender after removing debris. The weight of the ground material was $892.24 \mathrm{~g}$.

Five-hundred grams of the ground material were put in a 5-liter conical flask and $99.8 \%$ AR/ACS methanol (meets the standard Macron Fine Chemicals ${ }^{T M}$ grade of analytical reagents and the requirements of the American Chemical Society Committee on Analytical Reagents) added and stirred at room temperature for 24 hours for methanolic extraction. Whatman filter papers with particle retention of $>11 \mu \mathrm{m}$ and Welch 
0.9 CFM DryFast 2 Head PTFE diaphragm vacuum pump were used to filter the extract. The filtrate in a $250 \mathrm{~mL}$ round-bottomed flask was reduced in vacuum using a rotary evaporator and fixed using dry ice to form a uniform coating on the walls of the flask. It was then freeze-dried using a Heto PowerDryR LL1500 Freeze Dryer. For the aqueous extraction, $300 \mathrm{~g}$ of the ground material was mixed with $900 \mathrm{~mL}$ of distilled water in a conical flask and the mixture heated for $\mathbf{4 0}$ minutes. It was left overnight. It was then filtered, reduced, fixed, and freeze-dried for 24 hours like in the methanolic extraction process.

\section{Microbial Strains}

The $S$. aureus, S. pneumoniae, E. coli, and $C$. albicans were obtained from the stock of clinical isolates in the University of Nairobi's microbiology laboratory. S. pyogenes ATCC 19615 and MRSA ATCC 43300 were standard organisms obtained from the same laboratory. S. aureus, MRSA, and E. coli were provided while subcultured in nutrient agar. S. pyogenes and $S$. pneumoniae were provided while subcultured in blood agar. C. albicans was in Sabouraud's dextrose agar (SDA).

\section{Antimicrobial Susceptibility Testing}

Agar well diffusion method was used. Colonies from the subcultures were suspended in normal saline to attain an equivalent of McFarland's standard 0.5. Two milliliters of each of $S$. aureus, MRSA, and $E$. coli suspensions were added to their respective $200 \mathrm{~mL}$ of sterile trypticase soy agar (TSA) in liquid form at $50^{\circ} \mathrm{C}$. Suspensions of $S$. pyogenes and S. pneumoniae were similarly inoculated but in TSA with $5 \%$ defibrinated sheep blood. C. albicans was also inoculated following the same procedure but in SDA. The mixtures of media and suspension of the organisms were poured in $20 \mathrm{~mL}$ culture media plates. Eight plates were prepared for $S$. aureus, eight for $E$. coli, and six for each of the other four organisms. They were left to cool to room temperature. The plates were divided into two equal sets, one for testing the aqueous extracts and the other for the methanolic extracts.

An 8-mm sterile cork borer was used to punch five uniformly-spaced wells in each of the culture plates. The wells labeled $A, B, C, D$, and $E$ were for the negative control $(A)$, the positive control $(B)$, and 1000 $\mathrm{mg} / \mathrm{ml}(C), 100 \mathrm{mg} / \mathrm{ml}(D)$, and $10 \mathrm{mg} / \mathrm{ml}(E)$ concentrations of the extracts. A hundred microliters of distilled water for the negative control, $50 \mu \mathrm{L}$ of $0.3 \mathrm{mg} / \mathrm{ml}$ gentamicin (anti-bacterial) or $0.3 \mathrm{mg} / \mathrm{ml}$ nystatin (anti-fungal) for the positive control, and $100 \mu \mathrm{L}$ of each of the three doses of the aqueous and methanolic extracts were added in their respective wells. The plates were left at room temperature for one hour for the preparations to diffuse. The culture plates with S. aureus, MRSA E. coli, and C. albicans were incubated at room temperature for 18 hours. S. pyogenes and S. pneumoniae were incubated in $5 \% \mathrm{CO}_{2}$ at $37^{0} \mathrm{C}$ for 18 hours. Diameters of zones of inhibition were measured using a vernier caliper calibrated to give the measurements in a precision of two decimal points.

Mean diameter of zones of inhibition (mDZOI) was calculated for each concentration of the extracts by obtaining the average of the three or four measurements taken from the replicates of the experiments. $A$ $\mathrm{mDZOI}$ of $9.0 \mathrm{~mm}$ was used as the breakpoint, where $<9 \mathrm{~mm}$ was interpreted as resistance $(R)$ and $>9$ $\mathrm{mm}$ as susceptibility (S). The independent t-test was used to compare the mDZOI of each of the three 
dosages of Miraa extracts to the respective mDZOI of the respective negative control. One-way ANOVA was applied to determine whether at least one of the mDZOI was significantly different from the mean DZOI of other concentrations of Miraa extracts and the negative control. Tukey's HSD test was applied when ANOVA detected a significant difference so that it would be possible to know where the difference was lying. Paired t-test was used to compared the mDZOI of the aqueous Miraa extracts (aME) and the methanolic (mME). A 95\% confidence level was used in all the stages of data analysis. Level of significance of $5 \%$ and power of $80 \%$ were applied in all the analyses.

\section{Results}

\section{Extraction Yields}

The methanolic extraction process yielded $28.93 \mathrm{~g}$ from the $500 \mathrm{~g}$ ground material used, which was obtained from the Miraa leaves and twigs shown in figure 1. The yield's weight was $5.8 \%$ of the weight of the material used. On the other hand, the aqueous extraction method produced $6.75 \mathrm{~g}$ of extract from 300 $\mathrm{g}$ of the ground material. Thus, the extract's weight was $2.3 \%$ the weight of the material used.

\section{Antibacterial Effects against S. aureus}

Both aqueous Miraa extracts ( $\mathrm{aME}$ ) and methanol Miraa extracts (mME) significantly inhibited the growth of $S$. aureus at the three doses tested. The mDZOI for the $1000 \mathrm{mg} / \mathrm{ml}, 100 \mathrm{mg} / \mathrm{ml}$., and $10 \mathrm{mg} /$ $\mathrm{ml}$ concentrations of the aME and the corresponding positive and negative controls were as shown in figure 2 .

Independent t-test revealed the existence of a significant difference between the $\mathrm{mDZOI}$ of the 1000 $\mathrm{mg} / \mathrm{ml}, 100 \mathrm{mg} / \mathrm{ml}$, and $10 \mathrm{mg} / \mathrm{ml}$, and the negative control ( $p<0.05$ in all three comparisons). One-way ANOVA test showed that at least one of the mDZOls for the three doses was significantly different $(F=64.235, p=0.0001)$. Tukey HSD posthoc test identified the $\mathrm{mDZOI}$ of the $1000 \mathrm{mg} / \mathrm{ml}$ as the only one significantly different from the other two $(p=0.0001)$ for both tests.

For the $\mathrm{mME}$, each of the three doses had a significant antimicrobial effect against $S$. aureus. The $\mathrm{mDZOIs} \mathrm{were} \mathrm{as} \mathrm{shown} \mathrm{in} \mathrm{figure} 3$. They were all significantly different from the $\mathrm{mDZOI}$ of the negative control ( $p=0.0001$ for each of the concentrations). They were also significantly different from each other $(F=438.44, p=0.0001 ; p=0.0001$ for all sets of comparisons).

A paired t-test revealed that only the $10 \mathrm{mg} / \mathrm{ml}$ of the aME was different from the corresponding concentration of the $\mathrm{mME}(\mathrm{p}=0.001)$. The $1000 \mathrm{mg} / \mathrm{ml}$ and $100 \mathrm{mg} / \mathrm{ml}$ of aME had no significantly different effect compared to their respective concentrations of the mME.

\section{Antibacterial Effect of Miraa against MRSA ATCC 43300}

Both aME and mME had antibacterial effects against MRSA. The three concentrations of aME significantly inhibited the growth of MRSA. The mDZOIs are as shown in figure 4. They were all 
significantly different from the mDZOI of the negative control ( $\mathrm{p}<0.005$ for all the comparisons). Additionally, each of the three concentrations had an effect that was substantially different from the others $(F=400.387, P=0.0001 ; p=0.0001$ for all the sets of comparisons).

Similarly, the three concentrations of mME exerted significant inhibition against MRSA. The mDZOIs are shown in figure 5. Each of them had an mDZOI different from the negative control's $(p=0.0001)$. Their effects were significantly different from each other's $(F=214.884, p=0.0001 ; p<0.003$ for all the sets of comparisons). The aME exerted significantly greater effects compared to the $\mathrm{mME}$ at all three concentrations ( $p<0.003$ for all of them).

\section{Antibacterial Effects of Miraa Extracts against S. pneumoniae}

Both the aME and $\mathrm{mME}$ at $1000 \mathrm{mg} / \mathrm{ml}, 100 \mathrm{mg} / \mathrm{ml}$, and $10 \mathrm{mg} / \mathrm{ml}$ substantially inhibited the growth of the $S$. pneumoniae. For both the aME and $\mathrm{mME}$, there were significant differences between the mDZOIs of each of the concentrations of extracts and the respective negative control as shown in figure 6 and figure 7 ( $p=0.0001$ for all comparisons). There were also significant differences between the $\mathrm{mDZOI}$ of the three doses of $\operatorname{aME}(\mathrm{F}=1885.88, \mathrm{p}=0.0001 ; \mathrm{p}=0.0001$ for all sets of comparisons).

Similarly, the mDZOI of the three doses of $\mathrm{mME}$ were significantly different $(F=2714.72, P=0.0001$; $p=0.0001$ for all sets of comparisons). A comparison of the corresponding concentrations of aME and $\mathrm{mME}$ showed that only the mDZOls of the $10 \mathrm{mg} / \mathrm{ml}$ doses were significantly different $(p=0.01)$.

\section{Antibacterial Effect of Miraa Extracts against S. Pyogenes}

Both aME and mME significantly inhibited the growth of $S$. pyogenes. The mDZOI of each of the concentrations of aME and $\mathrm{mME}$ were as shown in figure 8 and 9 respectively. They were significantly different from the mDZOIs of the respective negative controls for both the aME and $\mathrm{mME}(p=0.0001)$.

The three doses of the aME had significantly different $m D Z O I(F=1568.6, P=0.0001 ; p=0.0001$ for all sets of comparisons). Similarly, the mME doses had mMDZOIs significantly different from each other $(F=926.76, p=0.0001 ; p=0.0001$ for all sets of comparisons. Comparison of the aME and $m M E$ revealed significant differences between the $100 \mathrm{mg} / \mathrm{ml}(p=0.009)$ and $10 \mathrm{mg} / \mathrm{ml}(p=0.013)$ doses.

Neither the aME nor mME inhibited the growth of E. coli and C. albicans.

\section{Discussion}

Few antimicrobial studies [8-12] have been done to investigate the antimicrobial properties of $C$. edulis. However, none of them indicated studying Miraa, the $C$. edulis cultivated in Kenya. The studied were conducted using C. edulis in Saudi Arabia [ 8], Yemen [9], United Kingdom [11], South Africa [12], and Lebanon [22]. The study by Al-hebshi (9), reported that different cultivars of $C$. edulis can have varying antimicrobial effects. Hence, it was critical to test whether Miraa, the $C$. edulis cultivars grown in Kenya, have antimicrobial effects like the cultivars tested in other countries. 
In the current study, the methanolic extraction process was more efficient than aqueous extraction. The yield from the methanolic extraction was $5.8 \% \mathrm{w} / \mathrm{w}$, which was more than double the $2.3 \% \mathrm{w} / \mathrm{w}$ yield of the aqueous extraction. None of the previous studies has reported comparisons of extraction yields; hence this finding could be novel.

Our findings on the antibacterial effects of Miraa against $S$. aureus are consistent with the ethnobiology findings by Kiunga et al.[14]. S. aureus is involved in multiple infections including the urogenital and gastrointestinal infections allegedly treated using Miraa in traditional medicine[14,16]. The results also agree with the study by Fatima et al. [8] which found both aqueous and methanolic extracts of Saudi Arabia's C.edulis to inhibit the growth of $S$. aureus clinical isolate at $0.125-1 \mathrm{mg} / \mathrm{ml}$. Further, McGaw et al. [12] found ethanol crude extracts of $C$. edulis in South Africa to have antimicrobial effects against $S$. aureus clinical isolate with a minimum inhibitory concentration of $0.012 \mathrm{mg} / \mathrm{ml}$. On the other hand, our findings contradict those of Al-hebshi's who found no growth inhibition with $1.25-20 \mathrm{mg} / \mathrm{ml}$ aqueous $C$. edulis in Yemen against S. aureus ATCC 6538 [9].

The difference in the activity of aME and $\mathrm{mME}$ at $10 \mathrm{mg} / \mathrm{ml}$, where the aME exhibited a stronger effect, could be due to the difference in composition of the extraction yields. Fatima et al. [8] demonstrated that aqueous and methanolic extracts of $C$. edulis have different constituent compounds. The antimicrobial effect against MRSA observed in the current study further points to the potential value of $C$. edulis in the search for alternatives to treat $S$. aureus infections. Possibly, the antimicrobial effect is due to $22 \mathrm{~b}$ hydroxytingenone and tingenone among other phytochemicals in the plant. Elhag et al.[10] extracted them from $C$. edulis callus cultures and found them to have antimicrobial effect against $S$. aureus at a $\mathrm{MIC}$ of $0.6 \mu \mathrm{g} / \mathrm{ml}$.

The results of the current study showing significant antimicrobial effects of Miraa against $S$. pyogenes are consistent with the findings by Kiunga et al.[14] that Miraa treats sore throat in traditional medicine. $S$. pyogenes cause sore throats and related sequelae[18]. The findings are also in agreement with those of Al-hebshi et al.[9], who found aqueous extracts of Yemen's $C$. edulis to have antimicrobial effects against S. pyogenes clinical isolate, MIC of $10-20 \mathrm{mg} / \mathrm{ml}$. The absence of effect observed by Fatima et al. [8] could be due to the low concentrations they tested, $0.125-1 \mathrm{mg} / \mathrm{ml}$. Al-hebshi et al. [9] also noted that at $1.25 \mathrm{mg} / \mathrm{ml}$, the aqueous extracts only stopped the hemolytic effect but not the growth of S. pyogenes. Therefore, the MIC of aqueous crude extracts of $C$. edulis against $S$. pyogenes could be between 1.25 $\mathrm{mg} / \mathrm{ml}$ and $10 \mathrm{mg} / \mathrm{ml}$.

In the study by Fatima et al. [8], methanolic extracts of $C$. edulis inhibited $S$. pyogenes at concentrations that aqueous extracts could not $(0.125-1 \mathrm{mg} / \mathrm{ml})$. The superior strength of the methanolic extracts against $S$. pyogenes was also observed in our study. The antimicrobial effect of the extracts is consistent despite the variability of the source of $C$. edulis cultivars (Kenya, Yemen, and Saudi Arabia) tested in the various research. The types of $S$. pyogenes used in the multiple studies are also diverse: standard organism, clinical isolate, and multi-drug resistant clinical isolate in: the current study, the research by Alhebshi et al.[9], and the study by Fatima et al.[8] respectively. 
The finding that Miraa extracts have substantial antimicrobial properties against $S$. pneumoniae is consistent with the folklore assertion that Miraa treats respiratory diseases [14]. S. pneumonia is a common cause of respiratory diseases [23]. This finding could be novel; we could not identify a publication of a previous study that reported the antimicrobial effects of $C$. edulis against $S$. pneumoniae. Given the rapidly-developing antimicrobial resistance against $S$. pneumoniae [19], our findings are valuable as they may aid the identification of sources of plant-based botanicals to use as alternatives to conventional antibiotics in treating S. pneumoniae infections.

Our findings of the absence of an antimicrobial effect of $C$. edulis against $E$. coli agree with the results of the study by Siddiqui et al.[11], in which methanolic extracts did not show inhibitory effects against the growth of E. coli K1 strain RS218 clinical isolate. Similarly, McGaw et al.[12] found ethanol extracts of $C$. edulis to have no antimicrobial effects against $E$. coli. Elhag et al. [10] also found no effect when they tested 22b-hydroxytingenone and tingenone compounds extracted from $C$. edulis callus culture against $E$. coli. Perhaps the urinary tract infections and gastrointestinal infections allegedly treated using Miraa in traditional medicine are caused by other pathogens such as $S$. aureus, but not E. coli [14].

In contrast, Fatima et al. [8] found $1 \mathrm{mg} / \mathrm{ml}$ of $C$. edulis extracts to inhibit growth of $E$. coli clinical isolate. However, the fact that $2.5 \%$ methanolic solution was used used to dilute their extracts instead of water as in the other studies may explain the variance in the results. Methanol is known to be antibacterial, but Fatima et al. [8] justified its use by indicating that is is not inhibitory at low concentrations.

Our findings that Miraa extracts have no antimicrobial effects against $C$. albicans are consistent with the findings by Al-hebshi et al. [9] and Elhag et al.[10]. It is unlikely that the oral and urogenital diseases treated by Miraa in traditional medicine are caused by $C$. albicans. Notably, just as in E. colis scenario, our findings on $C$. albicans differ from those of Fatima et al.[8], who found the extracts to inhibit the growth of $C$. albicans.

This research was derailed by a few limitations. First, scarcity of resources did not allow the purchase of standard organisms or characterization of the clinical isolates used. The use of available standard organisms and laboratory-stocked clinical isolates partly addressed the challenge. The inadequacy of resources also limited the number of pathogens included in the study and the varieties of extraction methods used. It was also not possible to use a quantitative method for the determination of MICs. The comparison of the effects of $C$. edulis from various countries was also done with caution because there is no study showing the variability of the genetic makeups of the $C$. edulis cultivars grown in various countries across the world.

\section{Conclusion}

In conclusion, the increasing emergence of antimicrobial resistance, the slowed-down discovery of new antibiotics, and the continued indiscriminate use of antibiotics pose a serious global challenge. The challenge can be partly addressed by exploring alternatives to conventional antibiotics for use in treating infectious diseases. Plant-based botanicals with antimicrobial properties are a suitable alternative. 
Investigating the antimicrobial effects of cultivated plants such as Miraa as potential plant-based botanicals is necessary because they show promise of consistent supply. This study showed that aqueous and methanolic crude Miraa extracts have antimicrobial effects against $S$. aureus, MRSA, $S$. pyogenes, and $S$. pneumoniae but not against $E$. coli and $C$. albicans. The revealed in-vitro antimicrobial effects provide the basis for testing the crude extracts in in vivo animal studies to help predict whether the crude extracts can have the observed antimicrobial effects in humans. Future studies should also identify the specific compounds in Miraa imparting the antimicrobial effect and assess the resistancemodifying effects of both the crude extracts and the isolated compounds.

\section{Declarations}

\section{Ethics approval and consent to participate}

Permission to study Catha edulis as part of MSc thesis was provided by Kenya Wildlife Service.

\section{Consent for publication}

Not applicable

\section{Availability of data and materials}

The dataset used and analyzed during the current study are available from the corresponding author on reasonable request.

\section{Competing interests}

The authors declare that they have no competing interests

\section{Funding}

The research cost was supported through the Centre of Materials, Product Development \& Nanotechnology (MAPRONANO)'s Master's Research Scholarship (Ksh. 200000, which is about \$2000).

\section{Author's Contributions}

DK designed the study, conducted the experiments, analyzed the data, and wrote the main draft of the manuscript.

SM was a major contributor in refining the manuscript.

$\mathrm{SN}$ was a major contributor in selecting the extraction methods and procedures

$\mathrm{HN}$ was integral in performing the extraction procedures and the antimicrobial susceptibility testing. 
JO provided substantial input in refining the research idea, selecting the microorganisms for the experiments, and choosing the antimicrobial susceptibility testing method and procedure.

All authors read and approved the final manuscript.

\section{Acknowledgements}

I acknowledge University of Nairobi for support with laboratory facilities and microorganisms for conducting the experiments.

I recognize my mother Sabina Nkatha, the herbalist who explained how she uses Miraa to treat some oral and genitourinary diseases.

I also acknowledge Gideon Thananga, Joseph Mwenda, and Stanley Mwenda for support in identifying the organic farm to purchase the Miraa needed for this study.

\section{Authors' Information}

DK is a final-year Master of Science student in the Department of Medical Microbiology and the Founder and Chief Executive Officer of MedRight Consulting LTD, a medical writing company.

SM is a Senior Lecturer in the Department of Conservative and Prosthetic Dentistry at the University of Nairobi.

SN is a Senior Lecturer of Pharmaceutical Chemistry in the Department of Pharmaceutical Chemistry at the University of Nairobi.

HM is a Pharmaceutical technologist in the Department of Pharmaceutical Chemistry at the University of Nairobi.

JO is an Associate Professor of Medical Microbiology in the Department of Medical Microbiology at the University of Nairobi.

\section{References}

1. Tandogdu Z, Wagenlehner FME. Global epidemiology of urinary tract infections. Current Opinion in Infectious Diseases. 2016; 1;29 (1):73-9

2. Gerbase AC, Zemouri C. Global Epidemiology of Sexually Transmitted Infections in the Twenty-First Century: Beyond the Numbers. In: Cristaudo A, Giuliani M. (eds) Sexually Transmitted Infections. Springer, Cham; 2020. p. 3-12.

3. Klein EY, Van Boeckel TP, Martinez EM, Pant S, Gandra S, Levin SA, et al. Global increase and geographic convergence in antibiotic consumption between 2000 and 2015. Proc Natl Acad Sci U S A. 2018; 10;115(15):E3463-70. 
4. Theuretzbacher U, Outterson K, Engel A, Karlén A. The global preclinical antibacterial pipeline. Nature Reviews Microbiology. 2020; 19;18(5):275-85.

5. Hollis A, Ahmed Z. Preserving antibiotics, rationally. N Engl J Med. 2013; 369:2474-6.

6. Gupta PD, Birdi TJ. Development of botanicals to combat antibiotic resistance. J Ayurveda Integr Med. 2017; 8(4):266-75.

7. Anand U, Jacobo-Herrera N, Altemimi A, Lakhssassi N. A comprehensive review on medicinal plants as antimicrobial therapeutics: Potential avenues of biocompatible drug discovery. Metabolites. 2019; 9(11):258.

8. Fatima N, Rizwan M, Hobani YH, Sayed A El, Kumar BV, Sunosi R Al, et al. Gas chromatography / Mass spectroscopy analysis of Catha edulis Forsk, A psycho stimulant revealed potent solvent dependent antimicrobial activity. J Pharmacogn PhytochemJ. 2017; 6(2):197-204.

9. Al-hebshi N, Al-haroni M, Skaug N. In vitro antimicrobial and resistance-modifying activities of aqueous crude khat extracts against oral microorganisms. Arch Oral Biol. 2006;51(3):183-8.

10. Elhag H, Mossa JS, El-olemy MM. Antimicrobial and Cytotoxic Activity of the Extracts of Khat Callus Cultures. Center for New Crops \& Plant Products, West Lafayette, Ind, USA. 1999;(Mic):463-6.

11. Siddiqui R. Antibacterial and Anti-Acanthamoebic Properties of Catha Edulis (Khat). J Bacteriol Parasitol. 2012; 3:7.

12. McGaw LJ, Jäger AK, Van Staden J. Antibacterial, anthelmintic and anti-amoebic activity in South African medicinal plants. J Ethnopharmacol. 2000; 72(1-2):247-63

13. Chhetri B, Ali N, Setzer W. A Survey of Chemical Compositions and Biological Activities of Yemeni Aromatic Medicinal Plants. Medicines. 2015;2(2):67-92.

14. Kiunga JK, Lukhoba CW, Dossaji SF, Yenesew A. Research Article A Survey of Traditional Medicinal Uses of Catha Edulis ( Celastraceae ) in Meru and Embu Counties of Kenya. University of Nairobi Repository. 2016;3(1):1-12.

15. Ghosh D. Quality issues of herbal medicines: internal and external factors. Int J Complement Altern Med. 2018; 11(1):67-9

16. Ondusko DS, Nolt D. Staphylococcus aureus. Pediatr Rev. 2018; 39 (6) 287-298

17. Lee AS, De Lencastre H, Garau J, Kluytmans J, Malhotra-Kumar S, Peschel A, et al. Methicillinresistant Staphylococcus aureus. Nat Rev Dis Prim. 2018; 4(1):1-23.

18. Reglinski M, Sriskandan S. Streptococcus pyogenes. In: Tang Y, Sussman M, Schwartzman, J. Molecular Medical Microbiology: Second Edition. Academic Press. 2014. p. 675-716.

19. Albrich WC, Monnet DL, Harbarth S. Antibiotic Selection Pressure and Resistance in Streptococcus pneumoniae and Streptococcus pyogenes. Emerg Infect Dis. 2004; 10(3):514.

20. Croxen MA, Law RJ, Scholz R, Keeney KM, Wlodarska M, Finlay BB. Recent advances in understanding enteric pathogenic Escherichia coli. Clinical Microbiology Reviews. 2013; 26(4):82280. 
21. Dadar M, Tiwari R, Karthik K, Chakraborty S, Shahali Y, Dhama K. Candida albicans - Biology, molecular characterization, pathogenicity, and advances in diagnosis and control - An update. Microbial Pathogenesis. 2018; 117:128-38.

22. Othman L, Sleiman A, Abdel-Massih RM. Antimicrobial activity of polyphenols and alkaloids in middle eastern plants. Front Microbiol. 2019; 10:911.

23. Weiser JN, Ferreira DM, Paton JC. Streptococcus pneumoniae: Transmission, colonization and invasion. Nature Reviews Microbiology. 2018; 16(6):355-67

\section{Figures}

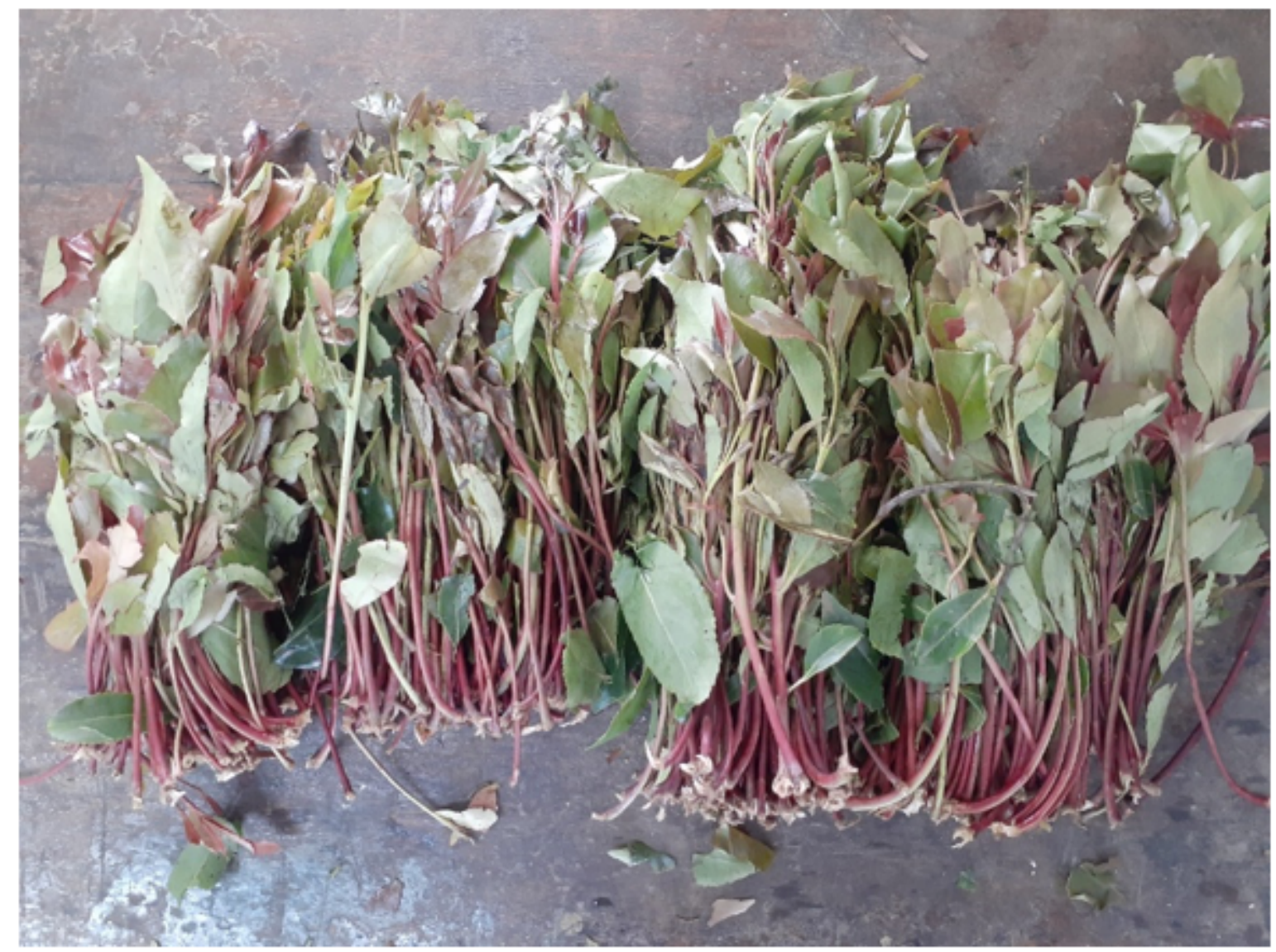

\section{Figure 1}

Miraa leaves and twigs before preparation for extraction 


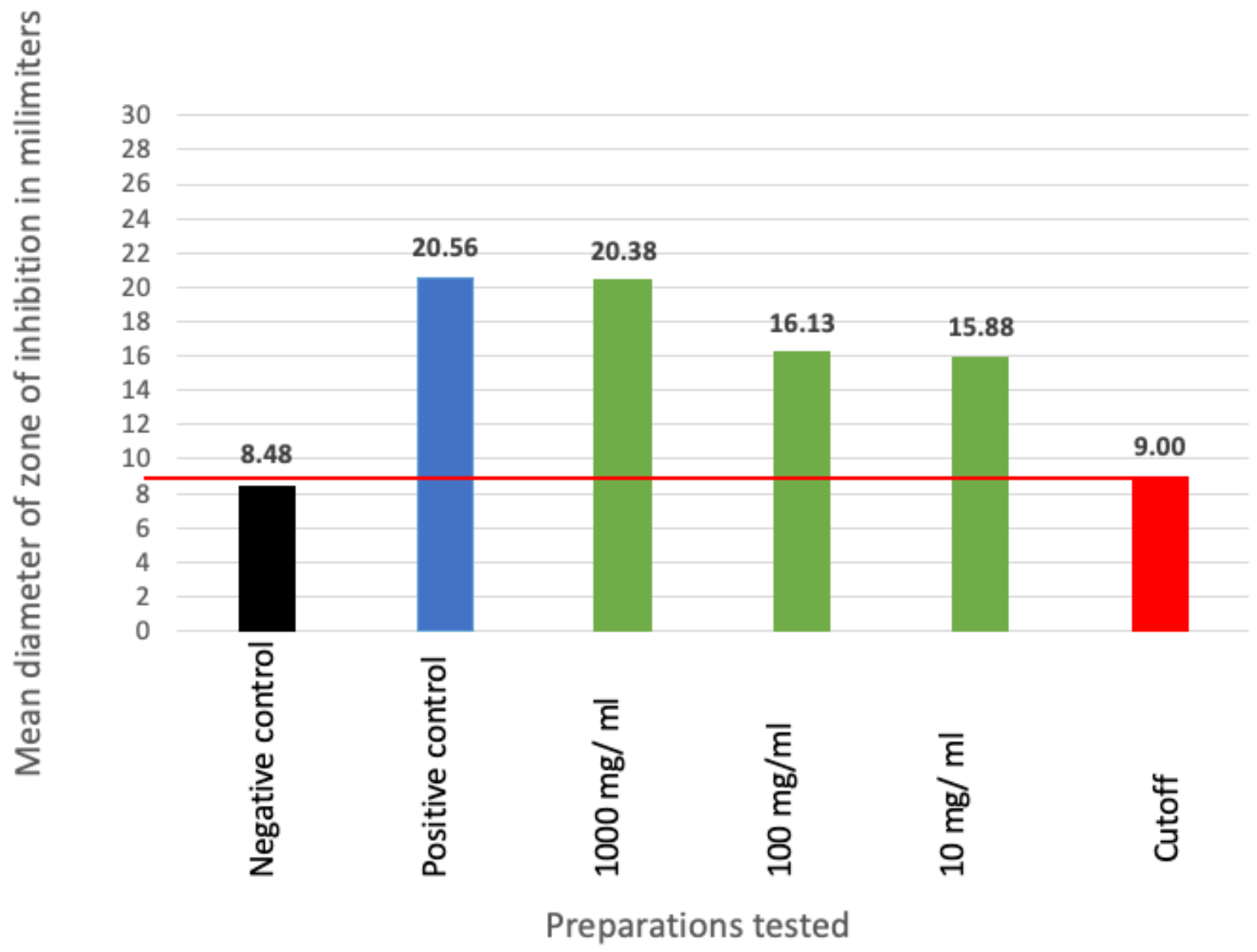

Figure 2

A graph showing the mean diameters of zone of inhibition of aqueous Miraa extracts against $\mathrm{S}$. aureus cultures 


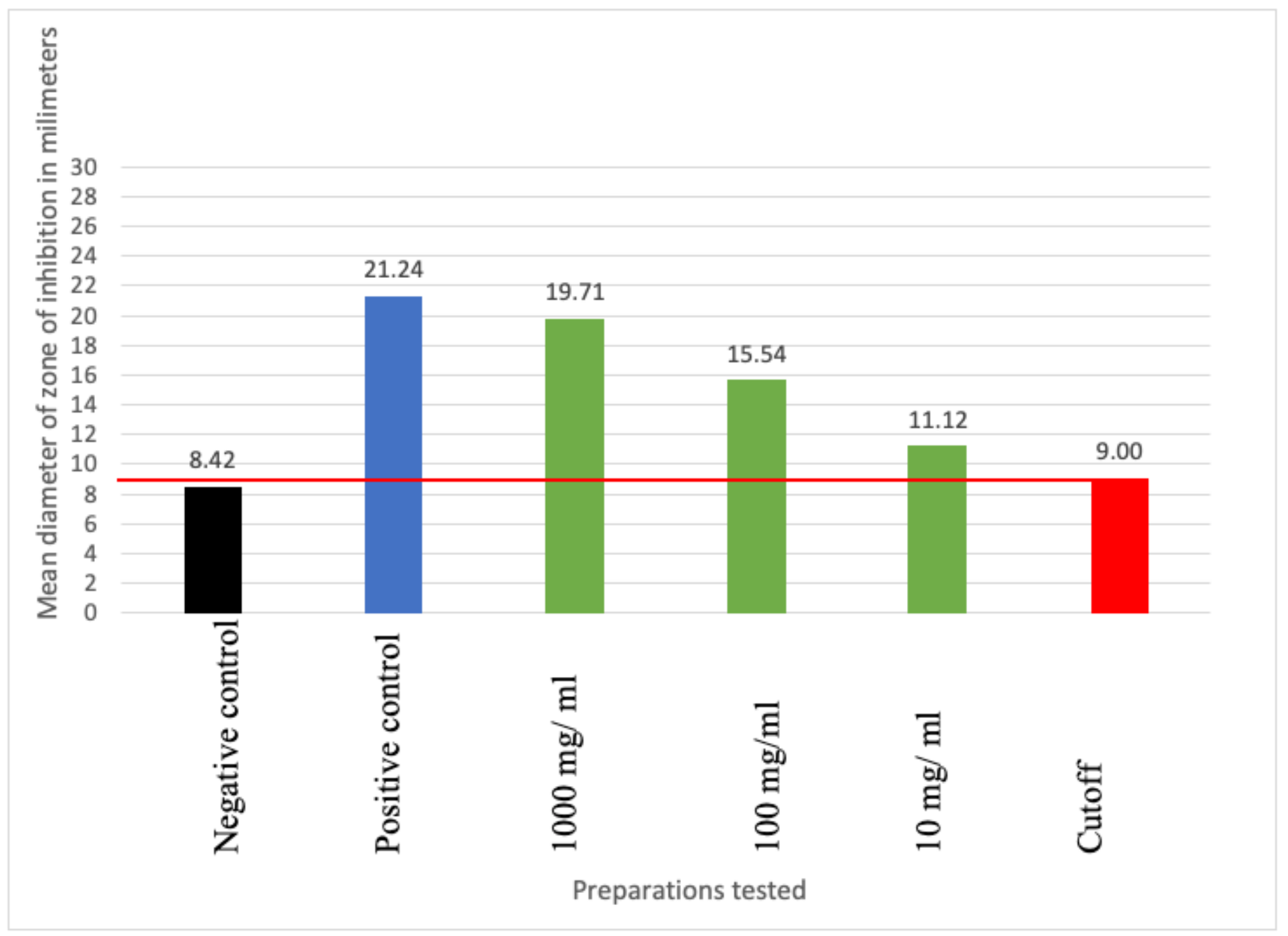

Figure 3

A graph showing the mean diameters of zone of inhibition of methanolic Miraa extracts against S. aureus cultures 


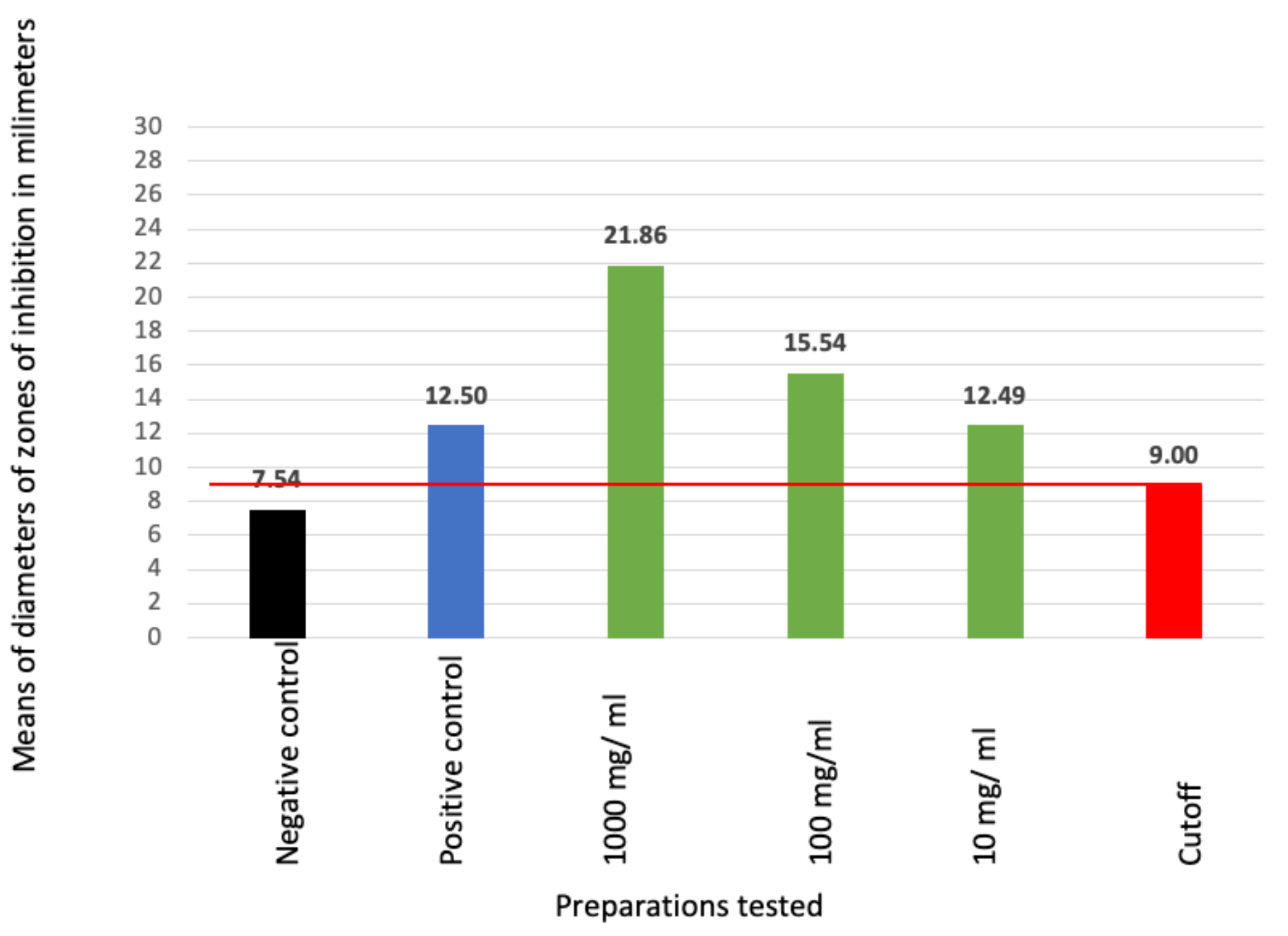

Figure 4

A graph showing the mean diameters of zone of inhibition of aqueous Miraa extracts against MRSA cultures 


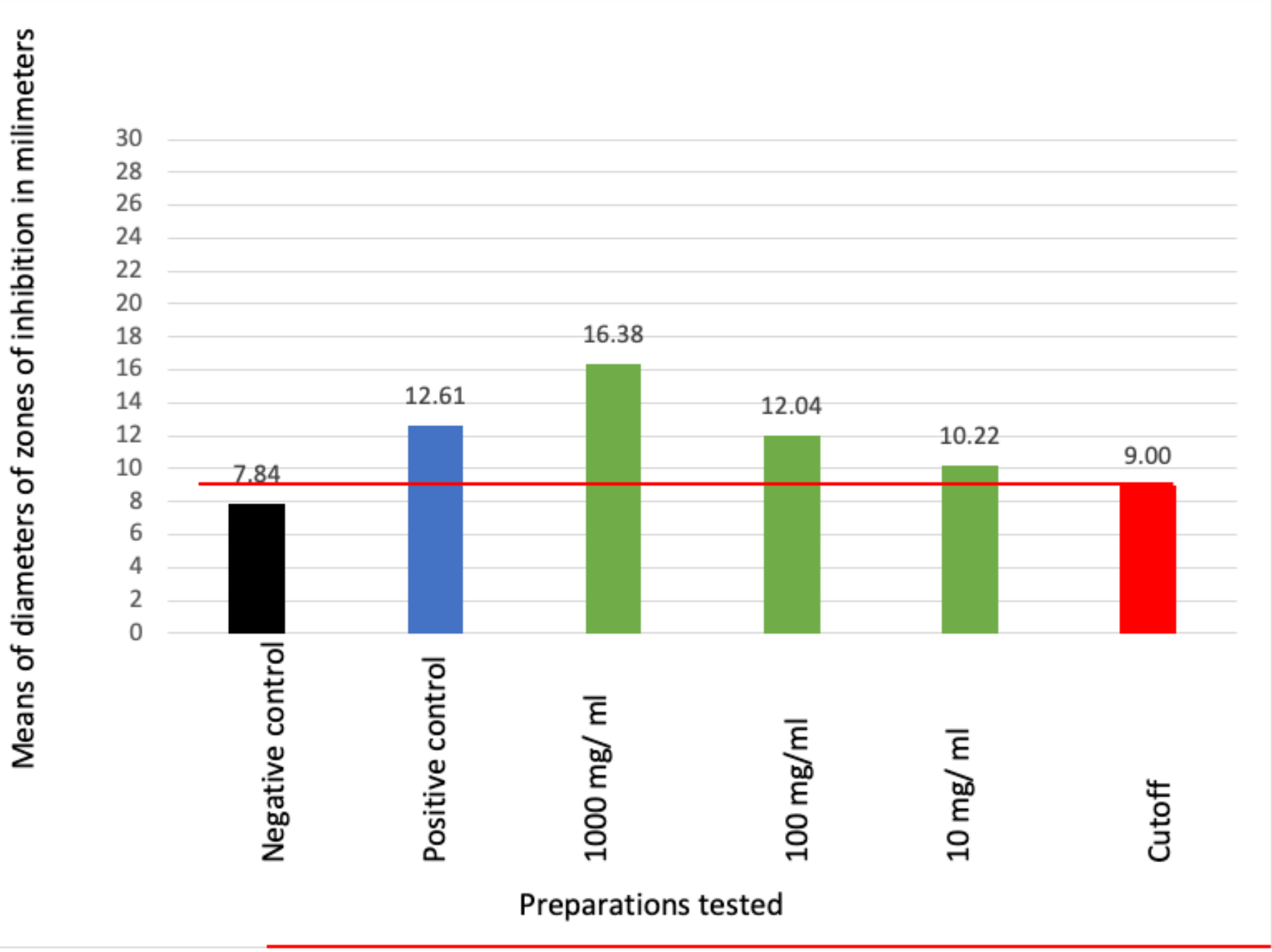

Figure 5

A graph showing the mean diameters of zone of inhibition caused by methanolic Miraa extracts in MRSA cultures 


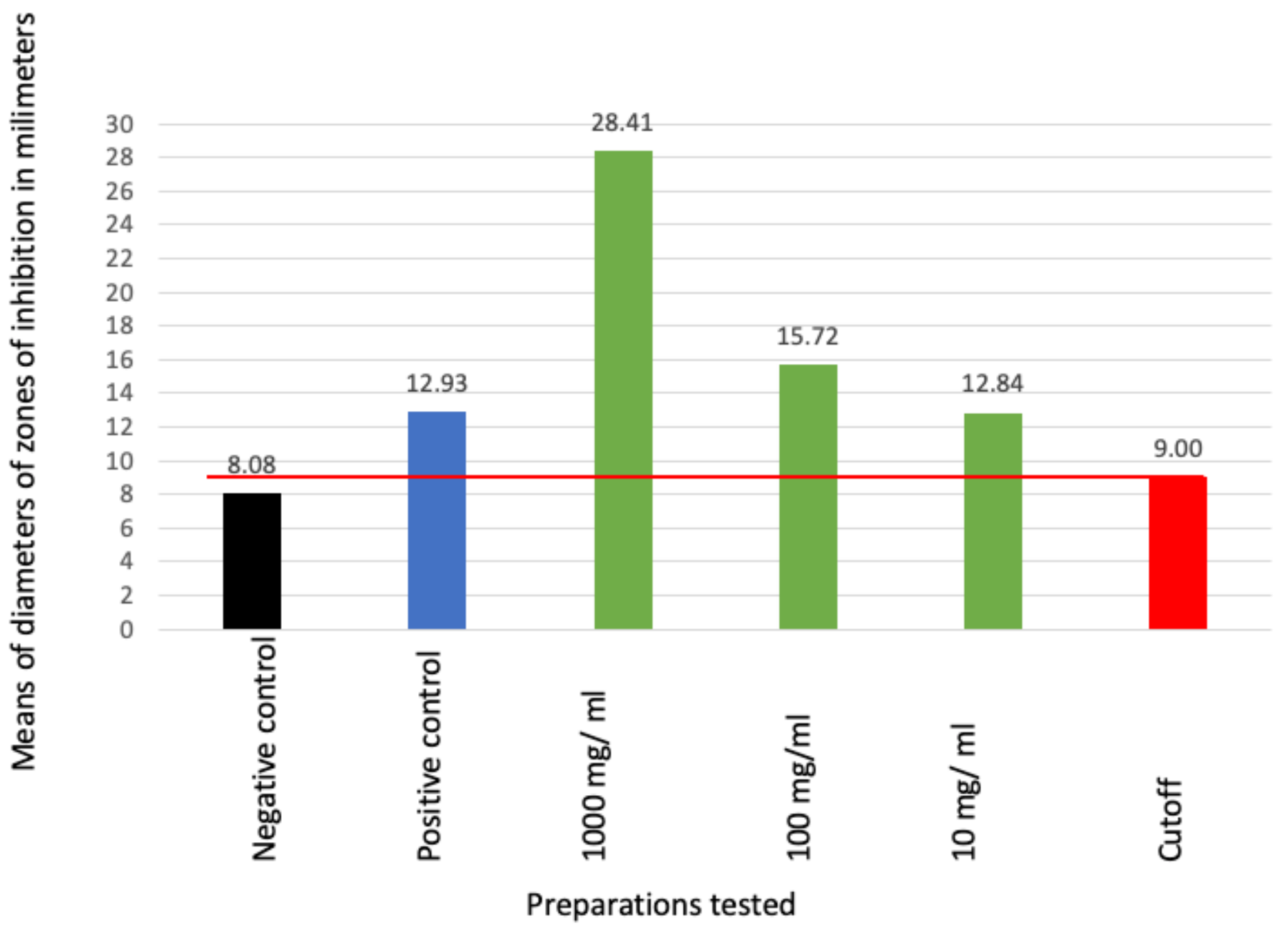

Figure 6

A graph showing the mean diameters of zone of inhibition caused by aqueous Miraa Extracts in Streptococcus pneumoniae cultures 


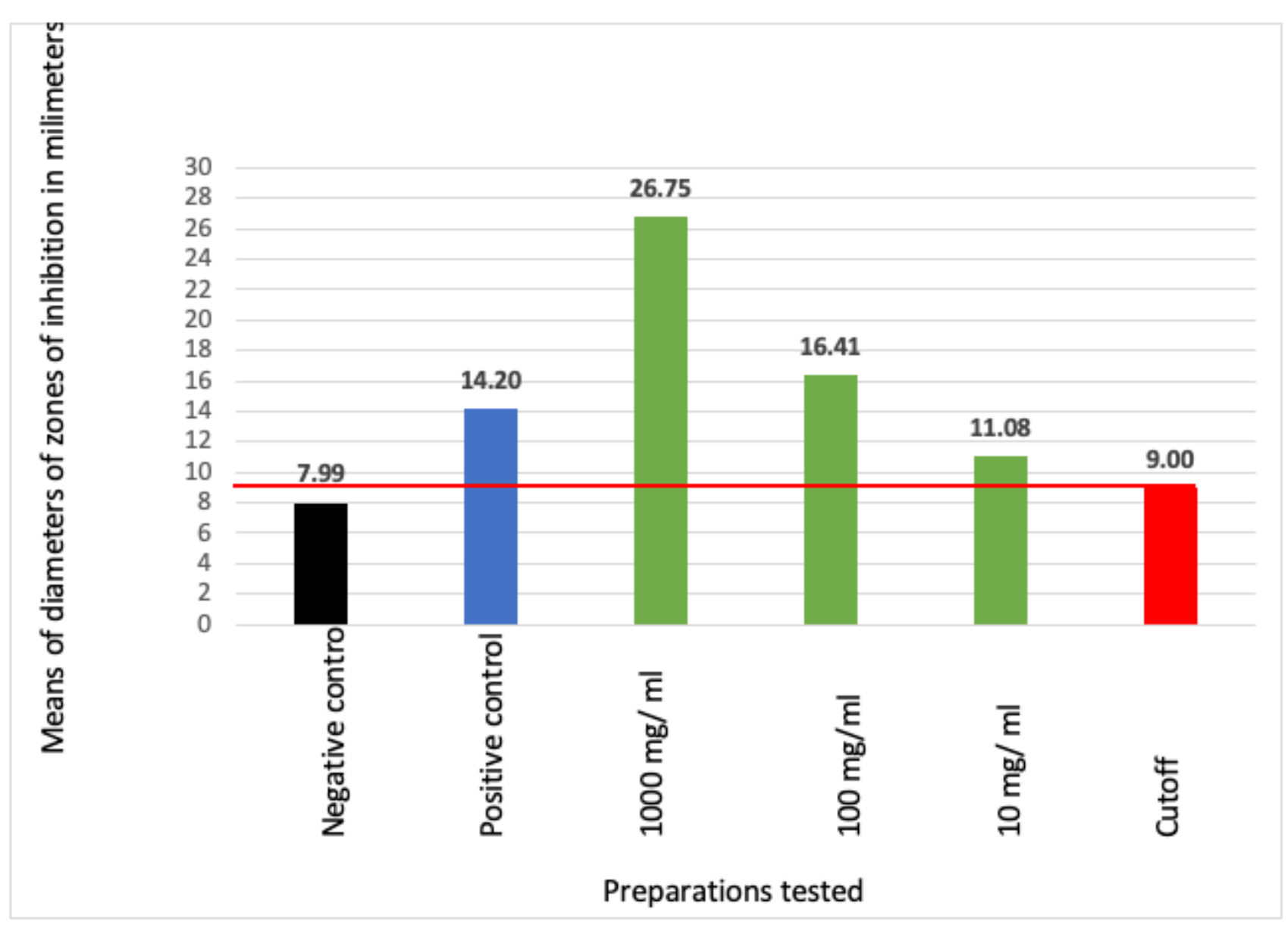

Figure 7

A graph showing the mean diameters of zone of inhibition caused by methanolic Miraa Extracts in Streptococcus pneumoniae cultures 


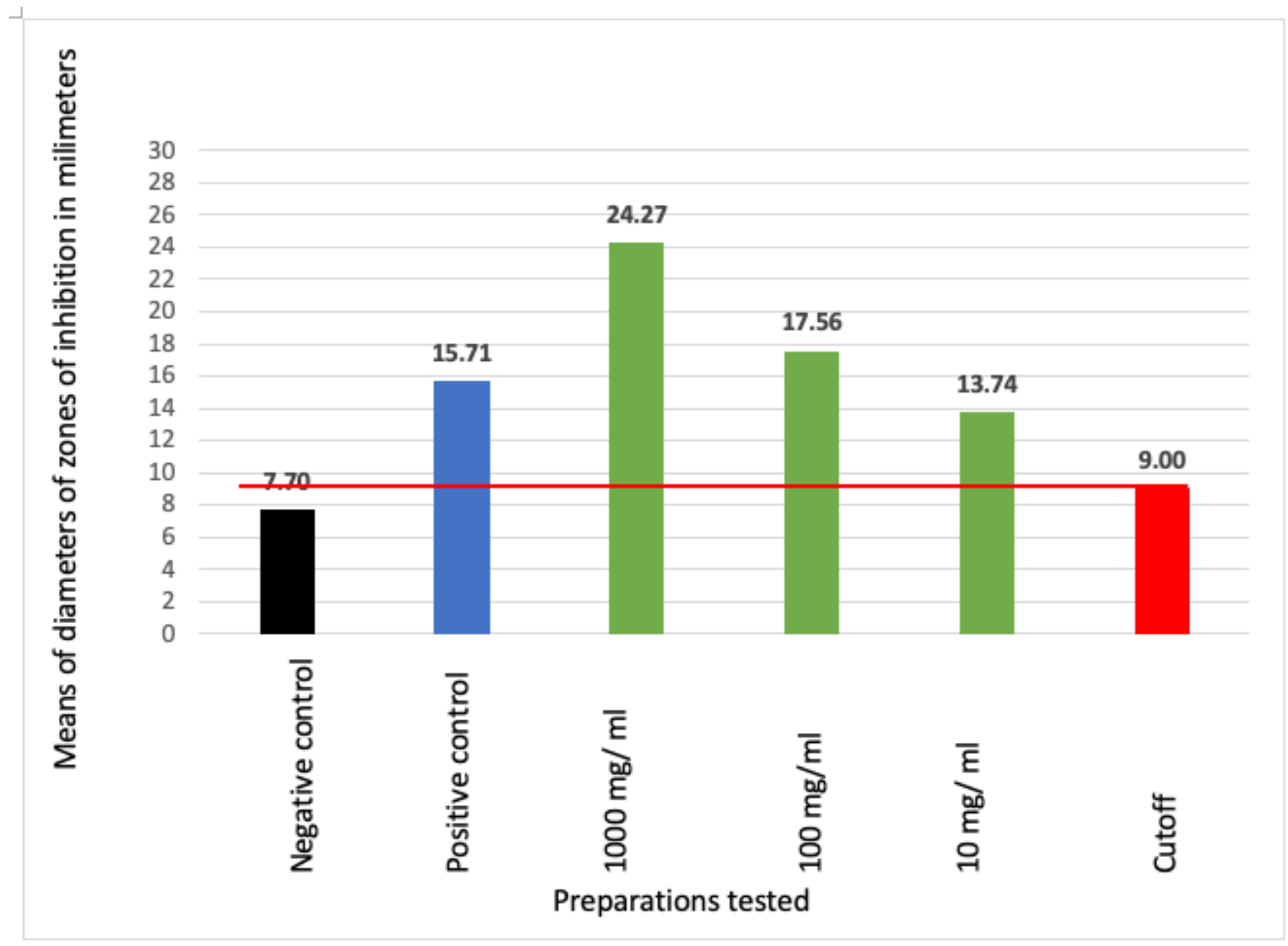

\section{Figure 8}

A graph showing the mean diameters of zone of inhibition caused by aqueous extracts of Miraa in Streptococcus pyogenes cultures 


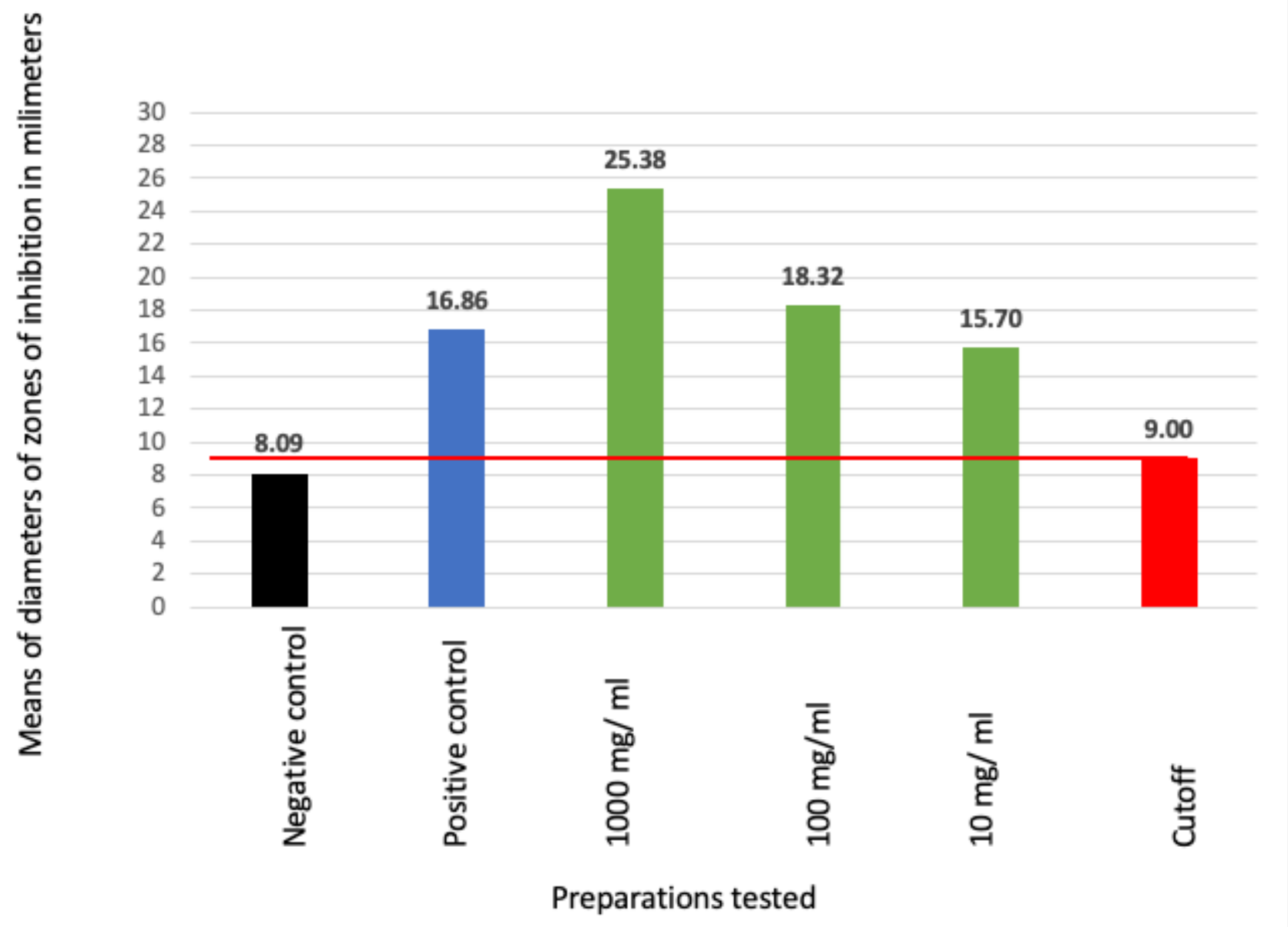

Figure 9

A graph showing the mean diameters of zone of inhibition caused by methanolic extracts of Miraa in Streptococcus pyogenes cultures 